\section{(C) OPEN ACCESS}

\title{
Stargardt disease: clinical features, molecular genetics, animal models and therapeutic options
}

\author{
Preena Tanna, ${ }^{1,2}$ Rupert W Strauss, ${ }^{1,2,3}$ Kaoru Fujinami, ${ }^{1,2,4}$ Michel Michaelides ${ }^{1,2}$
}

\begin{abstract}
${ }^{1} \mathrm{UCL}$ Institute of Ophthalmology, University College London, London, UK ${ }^{2}$ Moorfields Eye Hospital, London, UK

${ }^{3}$ Departments of Ophthalmology, Medical

University Graz and Johannes Kepler University, Linz, Austria

${ }^{4}$ National Institute of Sensory Organs, National Hospital

Organization, Tokyo Medical Centre, Tokyo, Japan
\end{abstract}

\section{Correspondence to} Michel Michaelides, UCL Institute of Ophthalmology, 11-43 Bath Street, London EC1V 9EL, UK; michel. michaelides@ucl.ac.uk

Received 4 April 2016 Revised 20 June 2016 Accepted 11 July 2016 Published Online First 4 August 2016

\section{CrossMark}

\footnotetext{
To cite: Tanna $P$, Strauss RW, Fujinami K, et al. Br J Ophthalmol 2017;101:25-30.
}

\begin{abstract}
Stargardt disease (STGD1; MIM 248200) is the most prevalent inherited macular dystrophy and is associated with disease-causing sequence variants in the gene $A B C A 4$. Significant advances have been made over the last 10 years in our understanding of both the clinical and molecular features of STGD1, and also the underlying pathophysiology, which has culminated in ongoing and planned human clinical trials of novel therapies. The aims of this review are to describe the detailed phenotypic and genotypic characteristics of the disease, conventional and novel imaging findings, current knowledge of animal models and pathogenesis, and the multiple avenues of intervention being explored.
\end{abstract}

\section{INTRODUCTION}

Stargardt disease (STGD1; MIM 248200) is the most common inherited macular dystrophy in both adults and children with a prevalence of 1 in 8000$10000 .^{1-7}$ STGD1 has an autosomal recessive mode of inheritance associated with disease-causing mutations in the $A B C A 4$ gene. ${ }^{8-11}$ It is both clinically and genetically highly heterogeneous. ${ }^{5-7} 12-17$

Patients present with bilateral central visual loss, including dyschromatopsia and central scotomata, with characteristic macular atrophy and yellowwhite flecks at the level of the retinal pigment epithelium (RPE) at the posterior pole. ${ }^{1} 5^{13} \quad 1819$ Onset is most commonly in childhood, with the next peak being early adulthood, and least frequently in later adulthood, with a better prognosis generally associated with a later onset. $^{2} 35713151619$ There is slow progressive loss of retinal function and structure over time; however, there is marked variability both within and between families, suggesting that other important factors influence phenotype, including genetic modifiers and the environment. ${ }^{12} 15162021$

Although there are currently no proven treatments, there are three main avenues of intervention being explored, with human clinical trials of stem cell therapy, gene replacement therapy and pharmacological approaches. $522-25$

\section{Clinical characteristics}

The characteristics of STGD1 vary widely due to the marked phenotypic heterogeneity associated with the large number $(>900)$ of disease-causing sequence variants identified in $A B C A 4 .^{26}$ There are various manifestations of the disease resulting in a spectrum of clinical presentations, rates of progression, imaging, psychophysical and electrophysiological findings, and variable prognosis.

STGD1 commonly presents as progressive bilateral central vision loss, with onset most often in childhood and a second peak incidence in early adulthood. ${ }^{3} 57$ There is increasing evidence that onset relates to the severity of the underlying ABCA4 variants, with childhood-onset STGD1 being associated with more deleterious variants (including nonsense variants) compared with adult-onset or the later onset 'foveal-sparing' (FS) STGD1 (more frequently, missense variants). $^{3} \quad 7 \quad 15 \quad 16 \quad 27$ Initially, ophthalmoscopy can reveal a normal fundus or mild retinal abnormalities (including loss of foveal reflex or mild RPE disturbance) with or without vision loss. ${ }^{5} 16$ The diagnosis can thereby be delayed unless retinal imaging with fundus autofluorescence (FAF) or spectral-domain optical coherence tomography (SD-OCT) and/or electrophysiological assessment (including pattern, full-field and multifocal electroretinography) are undertaken. ${ }^{3}$ SD-OCT will reveal loss of normal architecture that begins at the central macula with relative preservation of the peripheral macula in the first instance and reduced central autofluorescence surrounded by an increased signal or a bull's-eye maculopathy-like appearance on FAF. ${ }^{13} 2829$ It is important to note that up to a third of children at presentation may not have retinal flecks on fundoscopy or FAF these develop over time associated with increasing macular atrophy; another reason the diagnosis is often delayed is if retinal flecks are not present (figure 1). ${ }^{15}$ In very early childhood-onset disease with relatively preserved vision, symmetrical yellowish white fine dots at the central macula may also be seen. ${ }^{30} 31$

Although STGD1 is typically diagnosed in childhood or early adulthood, a later age of onset has increasingly been recognised. Late-onset STGD1 ('FS-STGD1') is a milder phenotype with a better prognosis and associated with foveal sparing. $^{15} \quad 16 \quad 19 \quad 32$ Patients with FS-STGD1 often have relatively preserved visual acuity and isolated macular dysfunction (normal full-field electroretinography (ERG)); yet still exhibit the wide phenotypic variability that is characteristic of $A B C A 4$-associated retinopathy, including rate of progression-with relative stability in some patients and more rapid progression in others. ${ }^{15} 1619$ Given that visual acuity may be relatively preserved, static automated perimetry is helpful in detecting and monitoring the pericentral ring-shaped scotoma that may impair reading ability in FS-STGD1. ${ }^{27}$ The mechanism(s) of foveal sparing is unknown; however, given that STGD1 is typically characterised by early foveal degeneration, this phenotype illustrates the potential influence of genetic and environmental factors on the mechanism of cell death; with evidence that specific missense variants 


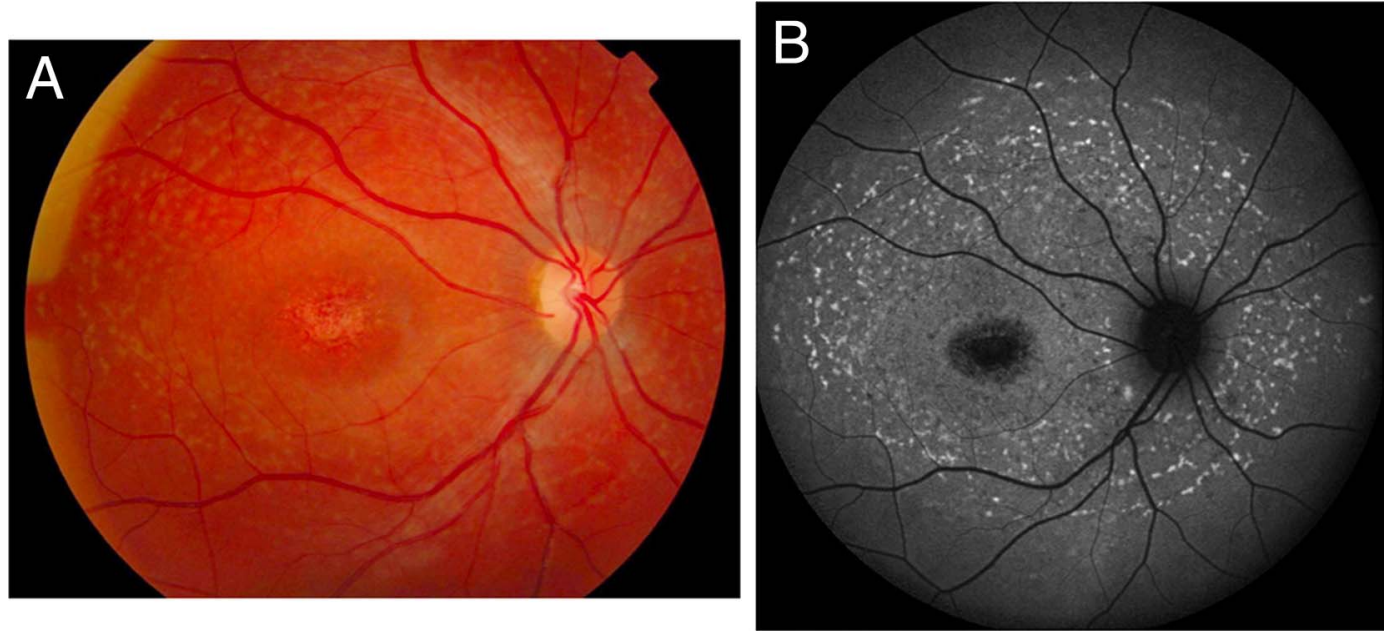

Figure 1 (A) Colour fundus photograph showing typical yellow-white retinal flecks with macular atrophy. (B) Corresponding fundus autofluorescence image showing flecks of both increased and decreased autofluorescence and reduced central macular autofluorescence surrounded by an increased signal.

may be more frequently observed in FS-STGD1 than non-FS phenotype. ${ }^{15}$ Individual foveal morphological factors such as cone density and macular pigment levels may also play a role in determining the FS phenotype. ${ }^{33} 34$

Electrophysiological assessment including multifocal, pattern and full-field electroretinography can be helpful in confirming the diagnosis of STGD1 and in providing better-informed advice on prognosis. Lois et $a l^{17}$ established a classification of three phenotypic subtypes of functional loss based on electrophysiological attributes. Group 1 is classified as a severe pattern ERG abnormality (macular dysfunction) with normal full-field ERGs; Group 2 is additional generalised loss of cone function; and Group 3 is additional generalised loss of both cone and rod function. ${ }^{17}$ A longitudinal study, incorporating the cohort of patients used in the aforementioned cross-sectional study to establish ERG group classifications, has now demonstrated that these groups have prognostic implications and that they do not reflect stages of disease; with Group 1 being associated with the best prognosis, Group 3 with the worst and Group 2 with an intermediate variable prognosis. ${ }^{2}$ A total of $22 \%$ of patients from Group 1 showed ERG group transition during follow-up, with $11 \%$ progressing to Group 2 and $11 \%$ to Group $3 .{ }^{2}$ Forty-seven per cent of patients in Group 2 progressed to Group $3 .^{2}$ There was clinically significant ERG deterioration in 54\% of all subjects: $22 \%$ of Group 1, 65\% of Group 2 and $100 \%$ of Group 3. Therefore, all patients with initial rod ERG involvement demonstrated clinically significant electrophysiological deterioration; only $20 \%$ of patients with normal fullfield ERGs showed clinically significant progression. ${ }^{2}$ Such data assist counselling by providing more accurate prognostic information (arguably the most accurate of all inherited retinal diseases) and are also relevant in the design, patient selection and monitoring of potential therapeutic interventions.

\section{Imaging and disease progression}

Imaging techniques providing enhanced assessment of retinal architecture have proven valuable in diagnosis, monitoring and probing the pathogenesis of STGD1, and include FAF, SD-OCT and adaptive optics scanning light ophthalmoscopy (AOSLO).

FAF imaging, by exploiting the autofluorescent properties of lipofuscin and related metabolites, affords assessment of RPE lipofuscin distribution in vivo. ${ }^{35} 36$ FAF has superseded fundus fluorescein angiography (FFA) in the diagnosis of STGD1, which was previously used to identify the 'dark choroid' observed in STGD1 due to blockage of underlying choroidal fluorescence by RPE-laden lipofuscin; ${ }^{7}$ though, not all patients have a dark choroid. ${ }^{18}$ Given that STGD1 is characterised by abnormal levels of lipofuscin, FAF imaging may identify fundus changes (early atrophy/flecks/dots) before they are clinically evident on ophthalmoscopy. ${ }^{3} 38$ An abnormally reduced FAF signal results from an absence or reduction in RPE lipofuscin density and/or RPE/photoreceptor cell loss. ${ }^{39}{ }^{40}$ In contrast, an abnormally increased FAF signal derives from excessive lipofuscin accumulation. ${ }^{40}$ Characteristic patterns of FAF, with areas of increased and decreased FAF, are observed in STGD1, and aid both diagnosis and measurement of progression over time. $^{5} 1341$ Quantified autofluorescence (qAF) has now been developed to indirectly measure RPE lipofuscin in vivo. ${ }^{42}$ Significantly elevated levels of qAF have been detected in STGD1 compared with healthy controls. ${ }^{42}$ A potential concern of qAF and conventional FAF is the use of short-wavelength light which may exacerbate the phototoxicity associated with $A B C A 4$-related disease. ${ }^{43}$ In response to this, Cideciyan et $a l^{44}$ have developed short-wavelength reduced-illuminance autofluorescence imaging that has been also implemented in the largest multicentre longitudinal study about the progression of atrophy secondary to STGD1. ${ }^{45}$

Several studies have retrospectively evaluated longitudinal FAF changes and patterns in STGD1. The largest published series to date $(n=68)$ classified patients into three FAF subtypes at baseline: type 1 had a localised low signal at the fovea surrounded by a homogeneous background $(n=19)$, type 2 had a localised low signal at the macula surrounded by a heterogeneous background with numerous foci of abnormal signal $(n=41)$ and type 3 had multiple low-signal areas at the posterior pole with a heterogeneous background $(n=8) .{ }^{13}$ The areas of reduced AF signal were measured, and the rate of atrophy enlargement (RAE) was calculated, with the mean follow-up interval being 9.1 years. The RAE $\left(\mathrm{mm}^{2} /\right.$ year) based upon baseline AF subtypes was significantly different: 0.06 in type $1,0.67$ in type 2 and 4.37 in type 3 . There was a significant association between AF subtype and genotype, with a preponderance of milder sequence variants (including missense) in type 1 and more deleterious sequence variants (including nonsense) being 
identified in type $3 .^{13}$ The observation that AF pattern at baseline influences the enlargement of atrophy over time and has genetic correlates assists in the provision of counselling on prognosis in STGD1 and is valuable for clinical trials.

SD-OCT provides high-resolution cross-sectional images of retinal lamination and allows early detection of foveal outer retinal loss, which is the hallmark of childhood- and adulthoodonset STGD1 (figure 2). The earliest OCT abnormality which has been detected in children, as young as 5 years of age, is external limiting membrane thickening, prior to the development of atrophy. ${ }^{30} 314647$ Serial OCT imaging may be employed to monitor progression by measuring changes in several parameters including total retinal thickness and macular volume, outer retinal thickness, outer nuclear layer (ONL) thickness and inner segment ellipsoid loss. ${ }^{28}$ For the purposes of clinical trial end-point validation, the demonstration of structure-function correlation will also likely be necessary. ${ }^{5}$ It is noteworthy that it has been shown that central foveal thickness on OCT correlates with visual acuity loss. ${ }^{27}$

AOSLO allows in vivo cellular imaging, with custom-built research systems now able to visualise rods, cones-including foveal cones-and RPE mosaics (figure 2). ${ }^{48}$ Both confocal and split-detector (SD; non-confocal) AOSLO imaging can be undertaken. $^{48}$ Confocal imaging requires relatively intact photoreceptor outer segment structure to detect wave-guiding photoreceptor cells. This thereby results in a significant number of 'dark spaces' observed in the cone mosaics of patients with
STGD1, where it was unknown until the advent of SD-AOSLO whether these dark spaces lacked an optical signal due to lack of cones or whether cones were present but not waveguiding. ${ }^{33} 474950$ This has fundamental implications for determining participant suitability and selection for intervention, and thereby clinical trial design and the likelihood of detecting safety and efficacy signals robustly and sensitively. In direct contrast, SD imaging does not require intact outer segments, but affords imaging of inner segments. The dark spaces have now been shown in many cases to harbour inner segments, although often 'swollen' compared with normal cone inner segments, suggesting there are more cones than previously imaged with confocal techniques that could potentially be rescued with novel therapies, including gene replacement. Moreover, SD imaging has identified cones that would not have been predicted on the basis of OCT alone, suggesting that $\mathrm{AO}$ imaging may be a more sensitive measure of photoreceptor integrity. ${ }^{48-50}$ However, these pilot observations need to be applied to large cohorts of molecularly proven patients over time, including quantitative AOSLO-derived measurements to determine disease progression on a cellular level over time, including foveal and parafoveal ('transition zone') cone densities and cone spacing. ${ }^{33} 47 \quad 49$ Structure-function correlations will also be valuable-with abnormal cone spacing having been previously shown to correlate to abnormal FAF and reduced visual function. ${ }^{47}$

The vast majority of data to date on disease progression is retrospective from single centres and thereby inherently limited.
Figure 2 Multimodal imaging of the right eye of a molecularly proven 15-year-old patient with Stargardt disease. (A) Fundus autofluorescence image. The yellow line indicates the scanning level of the optical coherence tomography (OCT) scan in (B). (B) OCT scan showing central loss of outer retinal structure. The yellow arrows indicate the location and extent of the adaptive optics scanning light ophthalmoscopy (AOSLO) montages in (C) and (D) through the transition zone. (C) Confocal AOSLO montage of the photoreceptor mosaic.

(D) Split-detection AOSLO montage of the photoreceptor mosaic. The far left side of (C) and (D) is closer to the fovea and lacks cone structure, corresponding with the lack of outer hyper-reflective layers in (B). Moving towards the right of (C) and (D), away from the fovea and superiorly on the retina, (C) shows structural changes that are relatively challenging to interpret, whereas (D) clearly shows the presence of cones. Scale bars represent $100 \mu \mathrm{m}{ }^{48}$
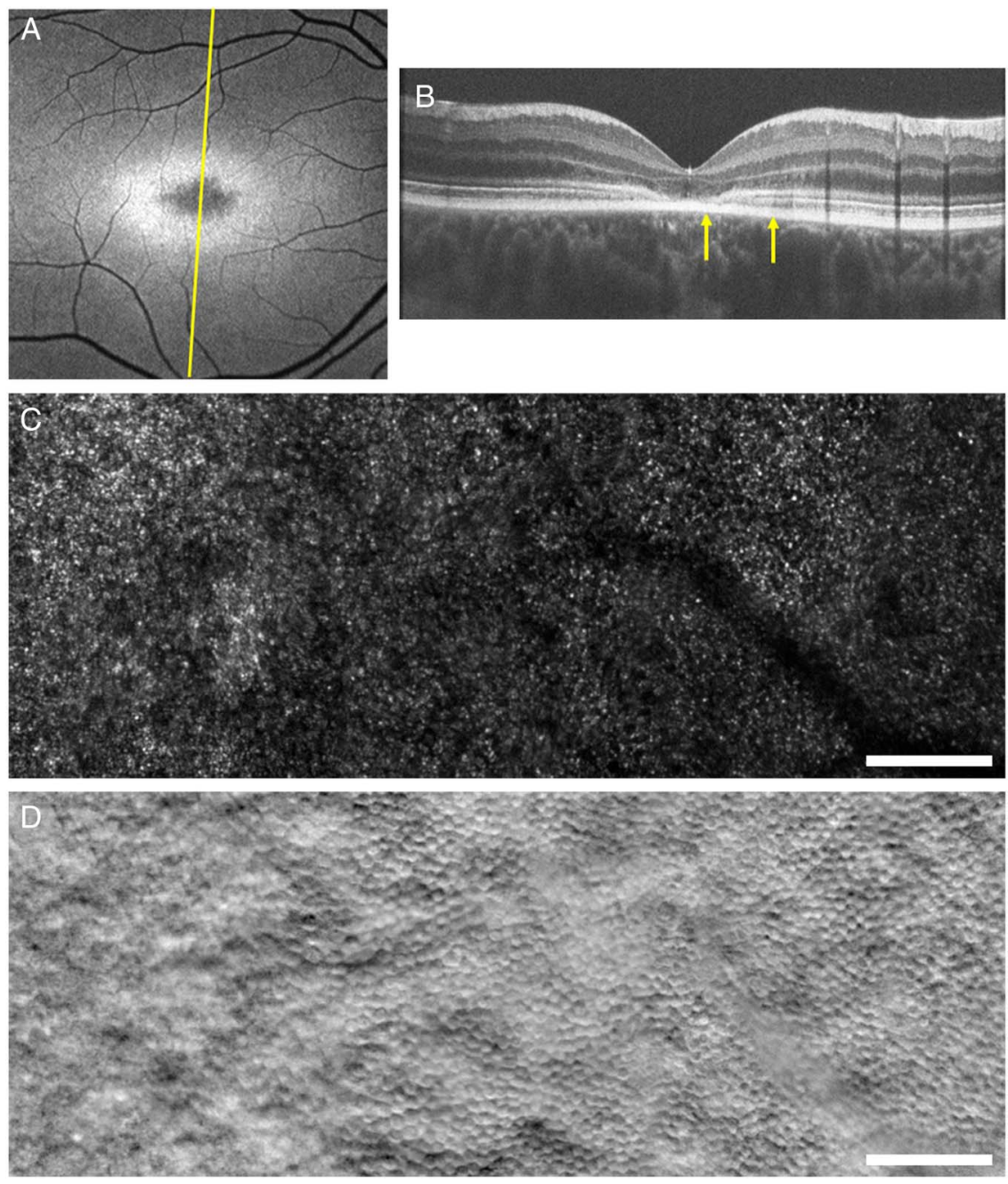
There is a need for prospective natural history studies of large cohorts of molecularly proven patients. One such ongoing multicentre international study is the 'Progression of Atrophy Secondary to Stargardt Disease (ProgStar)' study that characterises disease progression using psychophysical measures (such as mesopic and scotopic microperimetry) and imaging (SD-OCT and FAF) to establish endpoints for clinical trials of emerging therapies. 5

\section{Molecular genetics}

STGD1 is inherited in an autosomal recessive fashion and is caused by sequence variants in the gene $A B C A 4$, with the carrier frequency believed to be up to 1 in $20 .{ }^{51}$ Disease-causing variants in $A B C A 4$ are also associated with cone dystrophy, cone-rod and 'rod-cone' dystrophy. $A B C A 4$ is a large, highly polymorphic gene, consisting of 50 exons, with over 900 disease-associated variants reported to date. ${ }^{9} 10 \quad 26 \quad 52-54$ This highly polymorphic nature and large number of variants make ascribing definite disease-causation problematic; moreover, the vast allelic heterogeneity makes genotype-phenotype correlations very challenging indeed. In general, missense variants are associated with milder, later onset disease, while null alleles are associated with more severe, earlier onset disease; however, certain missense variants can also have severe functional effects similar to nulls (eg, p.Leu541Pro and p.Ala1038Val (complex), and p.Arg1640Trp). ${ }^{14} 5556$ The interaction between variants may also affect the functional outcome; while there is evidence that p.Gly1961Glu in the homozygous state typically causes a milder phenotype, a more severe phenotype results when associated with various additional $A B C A 4$ mutations. ${ }^{14} 57$ Certain missense variants appear to be more commonly observed in the mildest $A B C A 4$-associated phenotype, FS-STGD1, including p.Arg2030Gln. ${ }^{15} 58$

$A B C A 4$ is a member of the $\mathrm{ABC}$ transporter gene superfamily, encoding the retinal specific transmembrane ABCA4 protein, a member of the ATP-binding cassette transporter superfamily. ${ }^{59} 60$ ABCA4 is localised to the rim of the rod and cone outer segment discs, and is involved in the active transport of retinoids from photoreceptor to RPE. ${ }^{59-61}$

The high allelic heterogeneity makes molecular genetic testing of $A B C A 4$-associated retinal disease very challenging. It has been reported that direct Sanger sequencing of the entire $A B C A 4$ coding region detects between $66 \%$ and $80 \%$ of disease-causing alleles; however, this is not practically or economically possible in large cohorts. ${ }^{58} 62$ Arrayed primer extension (APEX) technology, which screens for all known previously reported $A B C A 4$ variants, detects approximately $65 \%$ to $75 \%$ of all disease-associated alleles. ${ }^{63}{ }^{64}$ However, by definition, novel variants are not detected by APEX technology, necessitating the use of other methodologies for high-throughput systematic screening of the entire coding region, especially in cases where one or both disease-causing alleles have failed to be identified by the array. A high-throughput strategy based on next-generation sequencing has now been shown to be highly effective in detecting both alleles, including new $A B C A 4$ variants not included in the APEX array. ${ }^{9} 10$ The identification of both disease-causing alleles improves the accuracy of diagnosis and the counselling of patients, and also assists in more effective patient selection of genetically confirmed participants for current and future clinical trials.

\section{Pathogenesis and animal models}

The visual cycle consists of enzyme-catalysed reactions converting all-trans retinal, derived from the photobleaching of rhodopsin and cone opsin, back to 11-cis retinal. ${ }^{60} 65$ All-trans retinal is released from the light-activated rhodopsin or cone opsin into the rod and cone outer segments, respectively, and forms a complex with phosphatidylethanolamine (PE) resulting in N-retinylidene-phosphatidylethanolamine (N-ret-PE), which is transported to the disc surface by ABCA4. The lack of, or inefficient removal of, N-ret-PE from photoreceptor outer segments, secondary to ABCA4 dysfunction/mislocalisation, results in an accumulation of bisretinoid compounds in outer segment discs, and ultimately in toxic levels of bisretinoid A2PE in photoreceptor membranes. ${ }^{436065} \mathrm{~A} 2 \mathrm{PE}$ is hydrolysed to form the highly toxic metabolite A2E, which accumulates as a component of lipofuscin in RPE cells, and ultimately results in RPE dysfunction and death, with subsequent photoreceptor dysfunction/loss. ${ }^{436667}$ While the commonly accepted RPE failure followed by subsequent photoreceptor cell dysfunction/death may be the sequence of events in the majority of $A B C A 4$-asociated disease, it may not be the case in, for example, FS-STGD1. ${ }^{15}$

While the STGD1 mouse model (ABCA4 knockout) has significant limitations, including that mice lack a macula (the primary area affected in STGD1) and the disease in mice is of later onset and exhibits slower degeneration than that seen in patients, it has helped shed light on the aforementioned underlying pathogenesis. Significantly elevated retinal levels of A2E and other lipofuscin fluorophores have been identified in this model. ${ }^{61}$ The ABCA4 knockout mice show delayed dark adaptation, increased all-trans-retinaldehyde following light exposure, increased PE in outer segments and accumulation of N-ret-PE. Moreover, they have an accelerated deposition of lipofuscin and $\mathrm{A} 2 \mathrm{E}$ in the RPE, supporting ABCA4's role as a transporter of N-ret-PE across disc membranes. ${ }^{68}$ The finding that the retinal degeneration in the knockout mouse was accelerated with exposure to significantly bright (ultraviolet) light ${ }^{69} 70$ and ingestion of large doses of vitamin $\mathrm{A}^{43}{ }^{71}$ has led to patients with STGD1 being advised to avoid excessive exposure to bright sunlight and wear good-quality sunglasses with UVA/UVB blocking properties, and to also avoid vitamin A supplementation. Interestingly, in a light exposure study, five patients with STGD1 who wore a black contact lens in one eye during waking hours for 12 months were observed to have less progression on FAF imaging in their study eye compared with the fellow eye. ${ }^{72}$

\section{Avenues of intervention}

STGD1 is currently subject to more clinical trials than any other inherited retinal disease, with gene replacement, stem cell therapy and pharmacological approaches.

Gene replacement therapy targets viable photoreceptors, with the underlying aim being to slow or prevent further retinal degeneration; so, the earlier the intervention the better. Adeno-associated virus (AAV) vectors have been the gene transfer system of choice for human gene therapy, including retinal diseases. $^{73} 74$ However, a major limitation is that $A B C A 4$ is larger than the current $\mathrm{AAV}$ vector capacity, a challenge that needs to be addressed for other genes that commonly cause inherited retinal disease, including $U S H 2 A .^{75}$ Subretinal injection of a lentivirus vector delivering $A B C A 4$ has therefore been developed, given the larger cargo capacity of lentiviruses, and is currently in an ongoing Phase I/II clinical trial (ClinicalTrials. gov Identifier: NCT01367444). There have been no safety concerns in the first three cohorts of subjects with relatively advanced disease, and no definite evidence of efficacy, with the final cohort with less severe disease now being recruited, with arguably more potential to show benefit. ${ }^{22} 2476$ 
In view of the fact that RPE cell dysfunction/loss is believed to precede photoreceptor cell dysfunction/loss in STGD1 and that RPE cells can be generated in the laboratory relatively easily, a Phase I/II stem cell therapy trial (ClinicalTrials.gov Identifier: NCT01469832) has been undertaken using human embryonic stem cell derived RPE cells transplanted subretinally in patients with severe advanced STGD1. ${ }^{23}$ There have been no safety concerns to date, and efficacy data are awaited. It remains possible that RPE cell replacement alone will be insufficient and that photoreceptor cells will need to also be transplanted.

There are many drugs that are already available or have been specifically developed that target different aspects of the visual cycle (vitamin A recycling pathway) and may thereby be potentially beneficial in slowing or stopping progression in STGD1. ${ }^{65}$ These include agents aimed at either (i) reducing the formation of toxic by-products of the visual cycle, by reducing delivery of vitamin A or inhibition of various enzymes participating in the visual cycle, or (ii) directly targeting toxic metabolites such as A2E. One of these drugs, which aims to allow normal visual cycle function and yet stop N-ret-PE and A2E formation, is a chemically modified vitamin A taken orally that will compete with dietary vitamin A, normally enter the visual cycle, but will not dimerise and therefore not exit the visual cycle to generate A2E. ${ }^{25} 7778$ This is currently being investigated in a Phase II clinical trial (ClinicalTrials.gov Identifier NCT02402660).

\section{CONCLUSIONS}

STGD1 is one of the most common causes of inherited childhood and adulthood visual impairment, with inherited retinal disease now being the most common cause of certifiable blindness in the working age population in England and Wales and the second most common in childhood. ${ }^{79}$ STGD1 is both phenotypically and genetically highly heterogeneous with significant advances having been made in our ability to identify the disease at the earliest stages, characterise clinical features that allow better-informed advice on prognosis, perform accurate rapid molecular genetic testing, and in our understanding of underlying disease mechanisms. These developments have allowed multiple clinical trials to be currently ongoing with many more, especially drug trials, anticipated over the next 5-10 years. Further robust longitudinal prospective natural history studies, probing genotype-phenotype and structurefunction associations, are crucial in order to provide improved prognostication and genetic counselling, as well as optimisation of clinical trial design, including identifying suitable participants, windows of opportunity and the most sensitive and reliable outcome metrics.

Contributors PT, RWS and KF contributed substantially to the conception and design of the work, drafted the work, approved the final version to be published and agreed to be accountable for all aspects of the work in ensuring that questions related to the accuracy or integrity of any part of the work are appropriately investigated and resolved. MM contributed substantially to the conception and design of the work, revised critically the work, approved the final version to be published and agreed to be accountable for all aspects of the work in ensuring that questions related to the accuracy or integrity of any part of the work are appropriately investigated and resolved.

Funding This work was supported by grants from the National Institute for Health Research Biomedical Research Centre at Moorfields Eye Hospital National Health Service Foundation Trust and UCL Institute of Ophthalmology, Fight For Sight (UK), The Macular Society (UK), Moorfields Eye Hospital Special Trustees, Moorfields Eye Charity, The Wellcome Trust (099173/Z/12/Z), the Foundation Fighting Blindness (USA) and Retinitis Pigmentosa Fighting Blindness. Professor Michel Michaelides is a recipient of an FFB Career Development Award. Dr Rupert Strauss is supported by the Austrian Science Fund (FWF; Project number: J 3383-B23) and the Foundation Fighting Blindness Clinical Research Institute (USA). KF is supported by an FFB
Clinical Research Fellowship Program Award, Grant-in-Aid for Young Scientists (A), The Ministry of Education, Culture, Sports, Science and Technology (Japan), and National Hospital Organization Network Research Fund (Japan).

Competing interests None declared.

Provenance and peer review Not commissioned; externally peer reviewed.

Open Access This is an Open Access article distributed in accordance with the terms of the Creative Commons Attribution (CC BY 4.0) license, which permits others to distribute, remix, adapt and build upon this work, for commercial use, provided the original work is properly cited. See: http://creativecommons.org/ licenses/by/4.0/

\section{REFERENCES}

1 Michaelides M, Hunt DM, Moore AT. The genetics of inherited macular dystrophies. J Med Genet 2003;40:641-50.

2 Fujinami K, Lois N, Davidson AE, et al. A longitudinal study of stargardt disease: clinical and electrophysiologic assessment, progression, and genotype correlations. Am J Ophthalmol 2013;155:1075-88.

3 Fujinami K, Zernant J, Chana RK, et al. Clinical and molecular characteristics of childhood-onset Stargardt disease. Ophthalmology 2015;122:326-34.

4 Molday RS. Insights into the molecular properties of ABCA4 and its role in the visual cycle and stargardt disease. Prog Mol Biol Trans/ Sci 2015;134:415-31.

5 Strauss RW, Ho A, Munoz B, et al. The natural history of the progression of atrophy secondary to Stargardt disease (ProgStar) studies: design and baseline characteristics: ProgStar Report No. 1. Ophthalmology 2016;123:817-28.

6 Burke TR, Tsang SH. Allelic and phenotypic heterogeneity in ABCA4 mutations. Ophthalmic Genet 2011;32:165-74.

7 Lambertus S, van Huet RA, Bax NM, et al. Early-onset stargardt disease: phenotypic and genotypic characteristics. Ophthalmology 2015;122:335-44.

8 Allikmets $\mathrm{R}$, Singh $\mathrm{N}$, Sun $\mathrm{H}$, et al. A photoreceptor cell-specific ATP-binding transporter gene $(A B C R)$ is mutated in recessive Stargardt macular dystrophy. Nat Genet 1997:15:236-46.

9 Fujinami K, Zernant J, Chana RK, et al. ABCA4 gene screening by next-generation sequencing in a British cohort. Invest Ophthalmol Vis Sci 2013;54:6662-74.

10 Zernant J, Schubert C, Im KM, et al. Analysis of the ABCA4 gene by next-generation sequencing. Invest Ophthalmol Vis Sci 2011;52:8479-87.

11 Haji Abdollahi S, Hirose T. Stargardt-Fundus flavimaculatus: recent advancements and treatment. Semin Ophthalmol 2013;28:372-6.

12 Michaelides M, Chen LL, Brantley MA Jr, et al. ABCA4 mutations and discordant ABCA4 alleles in patients and siblings with bull's-eye maculopathy. $\mathrm{Br} J$ Ophthalmol 2007;91:1650-5

13 Fujinami K, Lois N, Mukherjee R, et al. A longitudinal study of Stargardt disease: quantitative assessment of fundus autofluorescence, progression, and genotype correlations. Invest Ophthalmol Vis Sci 2013;54:8181-90.

14 Fujinami K, Sergouniotis PI, Davidson AE, et al. The clinical effect of homozygous ABCA4 alleles in 18 patients. Ophthalmology 2013;120:2324-31.

15 Fujinami K, Sergouniotis PI, Davidson AE, et al. Clinical and molecular analysis of Stargardt disease with preserved foveal structure and function. Am J Ophthalmol 2013;156:487-501 e1.

16 Westeneng-van Haaften SC, Boon CJ, Cremers FP, et al. Clinical and genetic characteristics of late-onset Stargardt's disease. Ophthalmology 2012;119: $1199-210$.

17 Lois N, Holder GE, Bunce C, et al. Phenotypic subtypes of Stargardt macular dystrophy-fundus flavimaculatus. Arch Ophthalmol 2001;119:359-69.

18 Fishman GA, Stone EM, Grover $\mathrm{S}$, et al. Variation of clinical expression in patients with Stargardt dystrophy and sequence variations in the ABCR gene. Arch Ophthalmol 1999;117:504-10.

19 Rotenstreich Y, Fishman GA, Anderson RJ. Visual acuity loss and clinical observations in a large series of patients with Stargardt disease. Ophthalmology 2003;110:1151-8.

20 Burke TR, Tsang SH, Zernant J, et al. Familial discordance in Stargardt disease. Mol Vis 2012;18:227-33.

21 Singh R, Fujinami K, Chen LL, et al. Longitudinal follow-up of siblings with a discordant Stargardt disease phenotype. Acta Ophthalmol 2014;92:e331-2.

22 Smith J, Ward D, Michaelides M, et al. New and emerging technologies for the treatment of inherited retinal diseases: a horizon scanning review. Eye (Lond) 2015;29:1131-40.

23 Schwartz SD, Regillo CD, Lam BL, et al. Human embryonic stem cell-derived retinal pigment epithelium in patients with age-related macular degeneration and Stargardt's macular dystrophy: follow-up of two open-label phase 1/2 studies. Lancet 2015;385:509-16.

24 Dalkara D, Goureau O, Marazova K, et al. Let there be light: gene and cell therapy for blindness. Hum Gene Ther 2016;27:134-47.

25 Saad L, Washington I. Can vitamin A be improved to prevent blindness due to age-related macular degeneration, Stargardt disease and other retinal dystrophies? Adv Exp Med Biol 2016;854:355-61. 
26 Zernant J, Xie YA, Ayuso C, et al. Analysis of the ABCA4 genomic locus in Stargardt disease. Hum Mol Genet 2014;23:6797-806.

27 van Huet RA, Bax NM, Westeneng-Van Haaften SC, et al. Foveal sparing in Stargardt disease. Invest Ophthalmol Vis Sci 2014;55:7467-78.

28 Strauss RW, Munoz B, Wolfson Y, et al. Assessment of estimated retinal atrophy progression in Stargardt macular dystrophy using spectral-domain optical coherence tomography. Br J Ophthalmol 2015;100:956-62.

29 Gomes NL, Greenstein VC, Carlson JN, et al. A comparison of fundus autofluorescence and retinal structure in patients with Stargardt disease. Invest Ophthalmol Vis Sci 2009;50:3953-9.

30 Fujinami K, Singh R, Carroll J, et al. Fine central macular dots associated with childhood-onset Stargardt disease. Acta Ophthalmol 2014;92:e157-9.

31 Lee W, Noupuu K, Oll M, et al. The external limiting membrane in early-onset Stargardt disease. Invest Ophthalmol Vis Sci 2014;55:6139-49.

32 Fujinami K, Akahori M, Fukui M, et al. Stargardt disease with preserved central vision: identification of a putative novel mutation in ATP-binding cassette transporter gene. Acta Ophthalmol 2011;89:e297-8.

33 Song $H$, Rossi EA, Latchney $L$, et al. Cone and rod loss in Stargardt disease revealed by adaptive optics scanning light ophthalmoscopy. JAMA Ophthalmol 2015;133:1198-203.

34 Aleman TS, Cideciyan AV, Windsor EA, et al. Macular pigment and lutein supplementation in ABCA4-associated retinal degenerations. Invest Ophthalmol Vis Sci 2007; 48:1319-29.

35 von Ruckmann A, Fitzke FW, Bird AC. Distribution of fundus autofluorescence with a scanning laser ophthalmoscope. Br J Ophthalmol 1995;79:407-12.

36 Delori FC, Dorey CK, Staurenghi G, et al. In vivo fluorescence of the ocular fundus exhibits retinal pigment epithelium lipofuscin characteristics. Invest Ophthalmol Vis Sci 1995:36:718-29.

37 Wabbels B, Demmler A, Paunescu K, et al. Fundus autofluorescence in children and teenagers with hereditary retinal diseases. Graefes Arch Clin Exp Ophthalmol 2006;244:36-45.

38 Boon CJ, Jeroen Klevering B, Keunen JE, et al. Fundus autofluorescence imaging of retinal dystrophies. Vision Res 2008;48:2569-77.

39 Lois N, Halfyard AS, Bird AC, et al. Fundus autofluorescence in Stargardt macular dystrophy-fundus flavimaculatus. Am J Ophthalmol 2004;138:55-63.

40 Smith RT, Gomes NL, Barile G, et al. Lipofuscin and autofluorescence metrics in progressive STGD. Invest Ophthalmol Vis Sci 2009;50:3907-14.

41 McBain VA, Townend J, Lois N. Progression of retinal pigment epithelial atrophy in stargardt disease. Am J Ophthalmol 2012;154:146-54.

42 Burke TR, Duncker T, Woods RL, et al. Quantitative fundus autofluorescence in recessive Stargardt disease. Invest Ophthalmol Vis Sci 2014;55:2841-52.

43 Sparrow JR, Gregory-Roberts E, Yamamoto K, et al. The bisretinoids of retinal pigment epithelium. Prog Retin Eye Res 2012;31:121-35.

44 Cideciyan AV, Swider M, Aleman TS, et al. Reduced-illuminance autofluorescence imaging in ABCA4-associated retinal degenerations. J Opt Soc Am A Opt Image Sci Vis 2007;24:1457-67.

45 Strauss RW, Munoz B, Jha A, et al. Comparison of short-wavelength reducedilluminance and conventional autofluorescence imaging in Stargardt Macular Dystrophy. Am J Ophthalmol 2016;168:269-78.

46 Burke TR, Yzer S, Zernant J, et al. Abnormality in the external limiting membrane in early Stargardt disease. Ophthalmic Genet 2013;34:75-7.

47 Chen Y, Ratnam K, Sundquist SM, et al. Cone photoreceptor abnormalities correlate with vision loss in patients with Stargardt disease. Invest Ophthalmol Vis Sci 2011;52:3281-92.

48 Scoles D, Sulai YN, Langlo CS, et al. In vivo imaging of human cone photoreceptor inner segments. Invest Ophthalmol Vis Sci 2014;55:4244-51.

49 Michaelides M, Zakka F, Aboshiha J, et al. High-resolution imaging in stargardt disease: preliminary observations in preparation for intervention [abstract]. Invest Ophthalmol Vis Sci 2014;55:5016.

50 Razeen MM, Cooper RF, Langlo CS, et al. Correlating photoreceptor mosaic structure to clinical findings in Stargardt disease. Trans/ Vis Sci Technol 2016;5:6.

51 Jaakson K, Zernant J, Kulm M, et al. Genotyping microarray (gene chip) for the ABCR (ABCA4) gene. Hum Mutat 2003:22:395-403.

52 Valverde $D$, Riveiro-Alvarez $R$, Bernal $S$, et al. Microarray-based mutation analysis of the ABCA4 gene in Spanish patients with Stargardt disease: evidence of a prevalent mutated allele. Mol Vis 2006;12:902-8.

53 Riveiro-Alvarez R, Lopez-Martinez MA, Zernant J, et al. Outcome of ABCA4 disease-associated alleles in autosomal recessive retinal dystrophies: retrospective analysis in 420 Spanish families. Ophthalmology 2013;120:2332-7.

54 Rosenberg T, Klie F, Garred P, et al. N965S is a common ABCA4 variant in Stargardt-related retinopathies in the Danish population. Mol Vis 2007;13:1962-9.
55 Wiszniewski W, Zaremba CM, Yatsenko AN, et al. ABCA4 mutations causing mislocalization are found frequently in patients with severe retinal dystrophies. Hum Mol Genet 2005;14:2769-78.

56 Briggs $C E$, Rucinski D, Rosenfeld PJ, et al. Mutations in ABCR (ABCA4) in patients with Stargardt macular degeneration or cone-rod degeneration. Invest Ophthalmol Vis Sci 2001;42:2229-36.

57 Burke TR, Fishman GA, Zernant J, et al. Retinal phenotypes in patients homozygous for the G1961E mutation in the ABCA4 gene. Invest Ophthalmol Vis Sci 2012;53:4458-67.

58 Yatsenko AN, Shroyer NF, Lewis RA, et al. Late-onset Stargardt disease is associated with missense mutations that map outside known functional regions of ABCR (ABCA4). Hum Genet 2001;108:346-55.

59 Cideciyan AV, Aleman TS, Swider M, et al. Mutations in ABCA4 result in accumulation of lipofuscin before slowing of the retinoid cycle: a reappraisal of the human disease sequence. Hum Mol Genet 2004;13:525-34.

60 Tsybovsky Y, Molday RS, Palczewski K. The ATP-binding cassette transporter ABCA4: structural and functional properties and role in retinal disease. Adv Exp Med Biol 2010;703:105-25.

61 Charbel Issa P, Barnard AR, Singh MS, et al. Fundus autofluorescence in the Abca4(-/-) mouse model of Stargardt disease--correlation with accumulation of $\mathrm{A} 2 \mathrm{E}$, retinal function, and histology. Invest Ophthalmol Vis Sci 2013;54:5602-12.

62 Shroyer NF, Lewis RA, Yatsenko AN, et al. Cosegregation and functional analysis of mutant $A B C R$ (ABCA4) alleles in families that manifest both Stargardt disease and age-related macular degeneration. Hum Mol Genet 2001;10:2671-8.

63 Ernest PJ, Boon CJ, Klevering BJ, et al. Outcome of ABCA4 microarray screening in routine clinical practice. Mol Vis 2009;15:2841-7.

64 Klevering BJ, Yzer S, Rohrschneider $\mathrm{K}$, et al. Microarray-based mutation analysis of the $A B C A 4(A B C R)$ gene in autosomal recessive cone-rod dystrophy and retinitis pigmentosa. Eur J Hum Genet 2004;12:1024-32.

65 Travis GH, Golczak M, Moise AR, et al. Diseases caused by defects in the visual cycle: retinoids as potential therapeutic agents. Annu Rev Pharmacol Toxicol 2007:47:469-512.

66 Sparrow JR, Boulton M. RPE lipofuscin and its role in retinal pathobiology. Exp Eye Res 2005:80:595-606.

67 Sparrow JR, Fishkin N, Zhou J, et al. A2E, a byproduct of the visual cycle. Vision Res 2003:43:2983-90.

68 Weng J, Mata NL, Azarian SM, et al. Insights into the function of Rim protein in photoreceptors and etiology of Stargardt's disease from the phenotype in abcr knockout mice. Cell 1999;98:13-23.

69 Chen Y, Okano K, Maeda T, et al. Mechanism of all-trans-retinal toxicity with implications for stargardt disease and age-related macular degeneration. J Biol Chem 2012:287:5059-69.

70 Radu RA, Mata NL, Bagla A, et al. Light exposure stimulates formation of A2E oxiranes in a mouse model of Stargardt's macular degeneration. Proc Natl Acad SCi USA 2004;101:5928-33.

71 Radu RA, Yuan Q, Hu J, et al. Accelerated accumulation of lipofuscin pigments in the RPE of a mouse model for ABCA4-mediated retinal dystrophies following Vitamin A supplementation. Invest Ophthalmol Vis Sci 2008;49:3821-9.

72 Teussink MM, Lee MD, Smith RT, et al. The effect of light deprivation in patients with Stargardt disease. Am J Ophthalmol 2015;159:964-72 e2.

73 Warrington KH Jr, Herzog RW. Treatment of human disease by adeno-associated viral gene transfer. Hum Genet 2006;119:571-603.

74 Allocca M, Doria M, Petrillo $M$, et al. Serotype-dependent packaging of large genes in adeno-associated viral vectors results in effective gene delivery in mice. J Clin Invest 2008:118:1955-64.

75 Han Z, Conley SM, Naash MI. Gene therapy for Stargardt disease associated with ABCA4 gene. Adv Exp Med Biol 2014;801:719-24.

76 Audo I, Weleber R, Stout T, et al. Early findings in a Phase I/lla clinical program for Stargardt disease (STGD1, MIM \#248200) [abstract]. Invest Ophthalmol Vis Sci 2015;56:3819.

77 Charbel Issa P, Barnard AR, Herrmann P, et al. Rescue of the Stargardt phenotype in Abca4 knockout mice through inhibition of vitamin A dimerization. Proc Natl Acad Sci USA 2015;112:8415-20.

78 Kaufman Y, Ma L, Washington I. Deuterium enrichment of vitamin A at the C20 position slows the formation of detrimental vitamin A dimers in wild-type rodents. J Biol Chem 2011;286:7958-65.

79 Liew G, Michaelides M, Bunce C. A comparison of the causes of blindness certifications in England and Wales in working age adults (16-64 years), 1999-2000 with 2009-2010. BMJ Open 2014;4:e004015. 